\title{
Energy dependence of commensurate neutron scattering peak in doped two-leg ladder antiferromagnet $\mathrm{Sr}_{14-x} \mathrm{Ca}_{x} \mathrm{Cu}_{24} \mathrm{O}_{41}$
}

\author{
Jianhui He and Shiping Feng \\ Department of Physics and Key Laboratory of Beam Technology and Material Modification, Beijing Normal University, \\ Beijing 100875, China \\ Interdisciplinary Center of Theoretical Studies, Chinese Academy of Sciences, Beijing 100080, China \\ National Laboratory of Superconductivity, Chinese Academy of Sciences, Beijing 100080, China \\ Wei Yeu Chen \\ Department of Physics, Tamkang University, Tamsui 25137, Taiwan
}

\begin{abstract}
The dynamical spin response of doped two-leg ladder antiferromagnets is investigated based on the fermion-spin approach. Our calculations clearly demonstrate a crossover from the incommensurate antiferromagnetism in the weak interchain coupling regime to commensurate spin fluctuation in the strong interchain coupling regime. In particular, the nuclear spin-lattice relaxation rate extracted from the commensurate spin fluctuation decreases exponentially with decreasing temperatures. The behaviors of the spin dynamics in the strong coupling regime are quantitatively close to the experimental results of $\mathrm{Sr}_{14-x} \mathrm{Ca}_{x} \mathrm{Cu}_{24} \mathrm{O}_{41}$.

71.27.+a, 74.72.-h, 76.60.-k
\end{abstract}

In recent years the novel two-leg ladder antiferromagnet $\mathrm{Sr}_{14} \mathrm{Cu}_{24} \mathrm{O}_{41}$, being situated between doped one- and twodimensional antiferromagnets, have been experimentally investigated as well as theoretically ${ }^{1}$. This followed from the fact that this material has a spin liquid ground $s^{2} \mathrm{te}^{2}$, which may play a crucial role in the superconductivity of doped cuprates as emphasized by Anderson ${ }^{3}$. Once carriers are added to the material $\mathrm{Sr}_{14} \mathrm{Cu}_{24} \mathrm{O}_{41}$, such as the isovalent substitution of $\mathrm{Ca}$ for $\mathrm{Sr}$, a metal-insulator transition has been observed ${ }^{1,4,5}$, and further, this doped two-leg ladder material $\mathrm{Sr}_{14-x} \mathrm{Ca}_{x} \mathrm{Cu}_{24} \mathrm{O}_{41}$ is a superconductor under pressure in low temperatures ${ }^{4,5}$. All cuprate superconductors found up now contain square $\mathrm{CuO}_{2}$ planes, whereas $\mathrm{Sr}_{14-x} \mathrm{Ca}_{x} \mathrm{Cu}_{24} \mathrm{O}_{41}$ consists of two-leg ladders of other $\mathrm{Cu}$ ions and edge-sharing $\mathrm{CuO}_{2}$ chains ${ }^{1,4,5}$, and is the only known superconducting copper oxide without a square lattice. Experimentally, it has been shown by virtue of transport measurements that there are the regions of parameter space where the resistivity is linear with temperatures ${ }^{5}$, one of the hallmarks of the exotic normal state properties found in the two-dimensional cuprates ${ }^{6}$. However, NMR and nuclear quadrupole resonance (NQR), particularly inelastic neutron scattering measurements in the same regions of parameter space indicate that $\mathrm{Sr}_{14-x} \mathrm{Ca}_{x} \mathrm{Cu}_{24} \mathrm{O}_{41}$ in the normal state is an antiferromagnet with the commensurate short-range order ${ }^{1,7,8}$. This commensurate spin correlation is energy dependent, and persists in a wide range of doping ${ }^{1,7,8}$. Moreover, NMR and NQR spin-lattice relaxation rate extracted from this commensurate spin fluctuation decreases exponentially with decreasing temperatures ${ }^{7,8}$. These magnetic behaviors are different from these of the doped two-dimensional high- $\mathrm{T}_{c}$ cuprates $^{9,10}$, where the incommensurate spin fluctuation for the single layer lanthanum cuprate ${ }^{9}$, and both low-energy incommensurate spin fluctuation and high-energy commensurate $[\pi, \pi]$ resonance for the bilayer yttrium cuprate ${ }^{10}$ in the normal state are observed.

Theoretically there is a general consensus that the unusual physical properties of the two-leg ladder materials are due to the quantum interference between the chains in the ladders ${ }^{1}$. Applying the bosonization procedure to the two-leg $t-J$ and Hubbard ladders, it is shown ${ }^{11}$ that all spin excitations are gapful and a singlet pairing becomes the dominant instability. These results are confirmed by some research groups within the different theoretical frameworks ${ }^{12}$. Furthermore, based on the antiferromagnetic Heisenberg model, the susceptibility and spin-lattice relaxation rate have been discussed ${ }^{13}$, and the results show that the large contribution to the spin-lattice relaxation rate comes from processes with the wave vectors around the antiferromagnetic zone center. Within the one dimensional gapped quantum nonlinear $\sigma$ model, an effective classical model has been developed to study the spin transport of the two-leg ladder antiferromagnets ${ }^{14}$, and the result obtained for the spin-lattice relaxation rate is close to what is experimentally measured. However, to the best of our knowledge, no systematic calculations have been performed to show why the commensurate neutron scattering peak can be observed in the doped two-leg ladder antiferromagnet $\mathrm{Sr}_{14-x} \mathrm{Ca}_{x} \mathrm{Cu}_{24} \mathrm{O}_{41}$ ? This is a challenge issue since it is closely related to the doped Mott insulating state that forms the basis for the superconductivity ${ }^{4}$. In this paper, using the fermion-spin theory ${ }^{15}$ which implements properly the local single occupancy constraint, we calculate explicitly the dynamical spin structure factor within a $t-J$ ladder and show that in the regions of parameter space given by the experiments, one can reproduce all main magnetic features observed experimentally on $\mathrm{Sr}_{14-x} \mathrm{Ca}_{x} \mathrm{Cu}_{24} \mathrm{O}_{41}$ in the normal state, including the energy dependence of the neutron scattering peak position and exponential decrease of the nuclear spin-lattice relaxation rate.

The two-leg ladder is defined as two parallel chains of ions, with bonds among them such that the interchain 
coupling is comparable in strength to the couplings along the chains, while the coupling between the two chains that participates in this structure is through rungs ${ }^{1,4}$. The essential properties of the doped two-leg ladder antiferromagnet can be described by the $t-J$ ladder as ${ }^{16}$,

$$
\begin{aligned}
H & =-t_{\|} \sum_{i \hat{\eta} a \sigma} C_{i a \sigma}^{\dagger} C_{i+\hat{\eta} a \sigma}-t_{\perp} \sum_{i \sigma}\left(C_{i 1 \sigma}^{\dagger} C_{i 2 \sigma}+\text { h.c. }\right) \\
& -\mu \sum_{i a \sigma} C_{i a \sigma}^{\dagger} C_{i a \sigma}+J_{\|} \sum_{i \hat{\eta} a} \mathbf{S}_{i a} \cdot \mathbf{S}_{i+\hat{\eta} a}+J_{\perp} \sum_{i} \mathbf{S}_{i 1} \cdot \mathbf{S}_{i 2},
\end{aligned}
$$

with the local constraint $\sum_{\sigma} C_{i a \sigma}^{\dagger} C_{i a \sigma} \leq 1$ to remove double occupancy of any site, where $\hat{\eta}= \pm c_{0} \hat{x}, c_{0}$ is the lattice constant of the two-leg ladder lattice, which is set as the unit hereafter, $i$ runs over all rungs, $\sigma(=\uparrow, \downarrow)$ and $a(=1,2)$ are spin and leg indices, respectively, $C_{i a \sigma}^{\dagger}\left(C_{i a \sigma}\right)$ are the electron creation (annihilation) operators, $\mathbf{S}_{i a}=C_{i a}^{\dagger} \vec{\sigma} C_{i a} / 2$ are the spin operators with $\vec{\sigma}=\left(\sigma_{x}, \sigma_{y}, \sigma_{z}\right)$ as the Pauli matrices, and $\mu$ is the chemical potential. For the two-leg ladder materials, it has been shown from experiments ${ }^{1,7,8}$ that the exchange coupling $J_{\|}$along the legs is greater than exchange coupling $J_{\perp}$ across a rung, i.e., $J_{\|}>J_{\perp}$, and similarly the hopping $t_{\|}$along the legs is greater than the rung hopping strength $t_{\perp}$, i.e., $t_{\|}>t_{\perp}$. On the other hand, the strong electron correlation in the $t$ - $J$ model is reflected by the local constraint ${ }^{3}$, which can be treated properly within the fermion-spin theory ${ }^{15}$, where electron operators $C_{i a \uparrow}=h_{i a}^{\dagger} S_{i a}^{-}$and $C_{i a \downarrow}=h_{i a}^{\dagger} S_{i a}^{+}$are represented by the spinless fermion operator $h_{i a}$ carrying the charge (holon) and the pseudospin operator $S_{i a}$ representing the spin (spinon), then it naturally incorporates the physics of charge-spin separation. In this case, the low-energy behavior of the $t$ - $J$ ladder (1) can be rewritten in the fermion-spin representation as,

$$
\begin{aligned}
H & =t_{\|} \sum_{i \hat{\eta} a} h_{i+\hat{\eta} a}^{\dagger} h_{i a}\left(S_{i a}^{+} S_{i+\hat{\eta} a}^{-}+S_{i a}^{-} S_{i+\hat{\eta} a}^{+}\right)+t_{\perp} \sum_{i}\left(h_{i 1}^{\dagger} h_{i 2}+h_{i 2}^{\dagger} h_{i 1}\right)\left(S_{i 1}^{+} S_{i 2}^{-}+S_{i 1}^{-} S_{i 2}^{+}\right) \\
& +\mu \sum_{i a} h_{i a}^{\dagger} h_{i a}+J_{\| \mathrm{eff}} \sum_{i \hat{\eta} a} \mathbf{S}_{i a} \cdot \mathbf{S}_{i+\hat{\eta} a}+J_{\perp \text { eff }} \sum_{i} \mathbf{S}_{i 1} \cdot \mathbf{S}_{i 2}
\end{aligned}
$$

where $J_{\| \text {eff }}=J_{\|}\left[(1-p)^{2}-\phi_{\|}^{2}\right], J_{\perp \text { eff }}=J\left[(1-p)^{2}-\phi_{\perp}^{2}\right], p$ is the hole doping concentration, the holon particle-hole order parameters $\phi_{\|}=\left\langle h_{i a}^{\dagger} h_{i+\hat{\eta} a}\right\rangle, \phi_{\perp}=\left\langle h_{i 1}^{\dagger} h_{i 2}\right\rangle$, and $S_{i a}^{+}\left(S_{i a}^{-}\right)$as the pseudospin raising (lowering) operators. It has been shown ${ }^{15}$ that the constrained electron operator can be mapped exactly using the fermion-spin transformation defined with an additional projection operator. However, this projection operator is cumbersome to handle in the actual calculations, and we have dropped it in Eq. (2) and in the subsequent calculation. It has also been shown ${ }^{15}$ that such treatment leads to errors of the order $p$ in counting the number of spin states, which is negligible for small dopings. In the two-leg ladder systems, because of the two coupled chains, the energy spectrum has two branches, therefore the one-particle spinon and holon Green's functions are matrices, and can be expressed as $D\left(i-j, \tau-\tau^{\prime}\right)=D_{L}(i-j, \tau-$ $\left.\tau^{\prime}\right)+\sigma_{x} D_{T}\left(i-j, \tau-\tau^{\prime}\right)$ and $g\left(i-j, \tau-\tau^{\prime}\right)=g_{L}\left(i-j, \tau-\tau^{\prime}\right)+\sigma_{x} g_{T}\left(i-j, \tau-\tau^{\prime}\right)$, respectively, where the longitudinal and transverse parts are defined as $D_{L}\left(i-j, \tau-\tau^{\prime}\right)=-\left\langle T_{\tau} S_{i a}^{+}(\tau) S_{j a}^{-}\left(\tau^{\prime}\right)\right\rangle, g_{L}\left(i-j, \tau-\tau^{\prime}\right)=-\left\langle T_{\tau} h_{i a}(\tau) h_{j a}^{\dagger}\left(\tau^{\prime}\right)\right\rangle$ and $D_{T}\left(i-j, \tau-\tau^{\prime}\right)=-\left\langle T_{\tau} S_{i a}^{+}(\tau) S_{j a^{\prime}}^{-}\left(\tau^{\prime}\right)\right\rangle, g_{T}\left(i-j, \tau-\tau^{\prime}\right)=-\left\langle T_{\tau} h_{i a}(\tau) h_{j a^{\prime}}^{\dagger}\left(\tau^{\prime}\right)\right\rangle$ with $a \neq a^{\prime}$. Within the chargespin separation, the spin fluctuation couples only to spinons ${ }^{17}$, however, the strong correlation between holons and spinons still is included self-consistently through the holon's order parameters entering the spinon's propagator, then both holons and spinons are responsible for the spin dynamics. In this case, the spin dynamics of the doped square lattice antiferromagnet have been discussed ${ }^{18}$, and the results are consistent with the experiments ${ }^{9,10}$. Following their discussions $^{18}$, the dynamical spin structure factor of the doped two-leg ladder antiferromagnet is obtained explicitly as,

$$
\begin{aligned}
S(\mathbf{k}, \omega) & =-2\left[1+n_{B}(\omega)\right]\left[2 \operatorname{Im} D_{L}(\mathbf{k}, \omega)+2 \operatorname{Im} D_{T}(\mathbf{k}, \omega)\right] \\
& =-\frac{4\left[1+n_{B}(\omega)\right]\left(B_{k}^{(1)}\right)^{2} \operatorname{Im} \Sigma_{L T}^{(s)}(\mathbf{k}, \omega)}{\left[\omega^{2}-\left(\omega_{k}^{(1)}\right)^{2}-B_{k}^{(1)} \operatorname{Re} \Sigma_{L T}^{(s)}(\mathbf{k}, \omega)\right]^{2}+\left[B_{k}^{(1)} \operatorname{Im} \Sigma_{L T}^{(s)}(\mathbf{k}, \omega)\right]^{2}},
\end{aligned}
$$

where the full spinon Green's function, $D^{-1}(\mathbf{k}, \omega)=D^{(0)-1}(\mathbf{k}, \omega)-\Sigma^{(s)}(\mathbf{k}, \omega)$, with the mean-field spinon Green's function,

$$
\begin{aligned}
D_{L}^{(0)}(\mathbf{k}, \omega) & =\frac{1}{2} \sum_{\nu} \frac{B_{k}^{(\nu)}}{\omega^{2}-\left(\omega_{k}^{(\nu)}\right)^{2}}, \\
D_{T}^{(0)}(\mathbf{k}, \omega) & =\frac{1}{2} \sum_{\nu}(-1)^{\nu+1} \frac{B_{k}^{(\nu)}}{\omega^{2}-\left(\omega_{k}^{(\nu)}\right)^{2}},
\end{aligned}
$$


$\nu=1,2, \quad \operatorname{Im} \Sigma_{L T}^{(s)}(\mathbf{k}, \omega)=\operatorname{Im} \Sigma_{L}^{(s)}(\mathbf{k}, \omega)+\operatorname{Im} \Sigma_{T}^{(s)}(\mathbf{k}, \omega), \quad \operatorname{Re} \Sigma_{L T}^{(s)}(\mathbf{k}, \omega)=\operatorname{Re} \Sigma_{L}^{(s)}(\mathbf{k}, \omega)+\operatorname{Re} \Sigma_{T}^{(s)}(\mathbf{k}, \omega)$, while $\operatorname{Im} \Sigma_{L}^{(s)}(\mathbf{k}, \omega)\left(\operatorname{Im} \Sigma_{T}^{(s)}(\mathbf{k}, \omega)\right)$ and $\operatorname{Re} \Sigma_{L}^{(s)}(\mathbf{k}, \omega)\left(\operatorname{Re} \Sigma_{T}^{(s)}(\mathbf{k}, \omega)\right)$ are the imaginary and real parts of the second order longitudinal (transverse) spinon self-energy, respectively, obtained from the holon bubble as $\Sigma_{L}^{(s)}(\mathbf{k}, \omega)=$ $(1 / L)^{2} \sum_{p p^{\prime}} \sum_{\nu \nu^{\prime} \nu^{\prime \prime}} \Pi_{\nu \nu^{\prime} \nu^{\prime \prime}}\left(\mathbf{k}, \mathbf{p}, \mathbf{p}^{\prime}, \omega\right)$ and $\Sigma_{T}^{(s)}(\mathbf{k}, \omega)=(1 / L)^{2} \sum_{p p^{\prime}} \sum_{\nu \nu^{\prime} \nu^{\prime \prime}}(-1)^{\nu+\nu^{\prime}+\nu^{\prime \prime}+1} \Pi_{\nu \nu^{\prime} \nu^{\prime \prime}}\left(\mathbf{k}, \mathbf{p}, \mathbf{p}^{\prime}, \omega\right)$ with $L$ is the number of rungs, and

$$
\begin{aligned}
\Pi_{\nu \nu^{\prime} \nu^{\prime \prime}}\left(\mathbf{k}, \mathbf{p}, \mathbf{p}^{\prime}, \omega\right) & =\left(2 t_{\|}\left[\cos \left(p^{\prime}+p+k\right)+\cos \left(p^{\prime}-k\right)\right]+t_{\perp}\left[(-1)^{\nu^{\prime}+\nu^{\prime \prime}}+(-1)^{\nu+\nu^{\prime \prime}}\right]\right)^{2} \\
& \times \frac{B_{k+p}^{\left(\nu^{\prime \prime}\right)}}{16 \omega_{k+p}^{\left(\nu^{\prime \prime}\right)}}\left(\frac{F_{\nu \nu^{\prime} \nu^{\prime \prime}}^{(1)}\left(\mathbf{k}, \mathbf{p}, \mathbf{p}^{\prime}\right)}{\omega+\xi_{p+p^{\prime}}^{\left(\nu^{\prime}\right)}-\xi_{p^{\prime}}^{(\nu)}-\omega_{k+p}^{\left(\nu^{\prime \prime}\right)}}-\frac{F_{\nu \nu^{\prime} \nu^{\prime \prime}}^{(2)}\left(\mathbf{k}, \mathbf{p}, \mathbf{p}^{\prime}\right)}{\omega+\xi_{p+p^{\prime}}^{\left(\nu^{\prime}\right)}-\xi_{p^{\prime}}^{\nu}+\omega_{k+p}^{\left(\nu^{\prime \prime}\right)}}\right)
\end{aligned}
$$

where $B_{k}^{(\nu)}=B_{k}-J_{\perp \text { eff }}\left[\chi_{\perp}+2 \chi_{\perp}^{z}(-1)^{\nu}\right]\left[\epsilon_{\perp}+(-1)^{\nu}\right], B_{k}=\lambda\left[\left(2 \epsilon_{\|} \chi_{\|}^{z}+\chi_{\|}\right) \cos k-\left(\epsilon_{\|} \chi_{\|}+2 \chi_{\|}^{z}\right)\right], \lambda=4 J_{\| \text {eff }}, \epsilon_{\|}=$ $1+2 t_{\|} \phi_{\|} / J_{\| \text {eff }}, \epsilon_{\perp}=1+4 t_{\perp} \phi_{\perp} / J_{\perp \text { eff }}$, and

$$
\begin{aligned}
& F_{\nu \nu^{\prime} \nu^{\prime \prime}}^{(1)}\left(\mathbf{k}, \mathbf{p}, \mathbf{p}^{\prime}\right)=n_{F}\left(\xi_{p+p^{\prime}}^{\left(\nu^{\prime}\right)}\left[1-n_{F}\left(\xi_{p^{\prime}}^{(\nu)}\right)\right]-n_{B}\left(\omega_{k+p}^{\left(\nu^{\prime \prime}\right)}\right)\left[n_{F}\left(\xi_{p^{\prime}}^{(\nu)}\right)-n_{F}\left(\xi_{p+p^{\prime}}^{\left(\nu^{\prime}\right)}\right)\right],\right. \\
& F_{\nu \nu^{\prime} \nu^{\prime \prime}}^{(2)}\left(\mathbf{k}, \mathbf{p}, \mathbf{p}^{\prime}\right)=n_{F}\left(\xi_{p+p^{\prime}}^{\left(\nu^{\prime}\right)}\left[1-n_{F}\left(\xi_{p^{\prime}}^{(\nu)}\right)\right]+\left[1+n_{B}\left(\omega_{k+p}^{\left(\nu^{\prime \prime}\right)}\right)\right]\left[n_{F}\left(\xi_{p^{\prime}}^{(\nu)}\right)-n_{F}\left(\xi_{p+p^{\prime}}^{\left(\nu^{\prime}\right)}\right)\right],\right.
\end{aligned}
$$

$n_{F}\left(\xi_{k}^{(\nu)}\right)$ and $n_{B}\left(\omega_{k}^{(\nu)}\right)$ are the fermion and boson distribution functions, respectively, the mean-field holon excitations $\xi_{k}^{(\nu)}=4 t_{\|} \chi_{\|} \cos k+\mu+2 \chi_{\perp} t_{\perp}(-1)^{\nu+1}$, and the mean-field spinon excitations, $\left(\omega_{k}^{(\nu)}\right)^{2}=\omega_{k}^{2}+\Delta_{k}^{2}(-1)^{\nu+1}$ with $\omega_{k}^{2}=A_{1}(\cos k)^{2}+A_{2} \cos k+A_{3}, \Delta_{k}^{2}=X_{1} \cos k+X_{2}$, where

$$
\begin{aligned}
A_{1} & =\alpha \epsilon_{\|} \lambda^{2}\left(\chi_{\|} / 2+\epsilon_{\|} \chi_{\|}^{z}\right), \\
A_{2} & =-\epsilon_{\|} \lambda^{2}\left[\alpha\left(\epsilon_{\|} \chi_{\|} / 2+\chi_{\|}^{z}\right) / 2-\alpha\left(C_{\|}^{z}+C_{\|} / 2\right)-(1-\alpha) / 4\right] \\
& -\alpha \lambda J_{\perp \text { eff }}\left[\epsilon_{\|}\left(C_{\perp}^{z}+\chi_{\perp}^{z}\right)+\epsilon_{\perp}\left(C_{\perp}+\epsilon \chi_{\perp}\right) / 2\right], \\
A_{3} & =\lambda^{2}\left[\alpha\left(C_{\|}^{z}+\epsilon_{\|}^{2} C_{\|} / 2\right)+(1-\alpha)\left(1+\epsilon_{\|}^{2}\right) / 8-\alpha \epsilon_{\|}\left(\chi_{\|} / 2+\epsilon_{\|} \chi_{\|}^{z}\right) / 2\right] \\
& +\alpha \lambda J_{\perp \text { eff }}\left[\epsilon_{\|} \epsilon_{\perp} C_{\perp}+2 C_{\perp}^{z}\right]+J_{\perp \text { eff }}^{2}\left(\epsilon_{\perp}^{2}+1\right) / 4, \\
X_{1} & =\alpha \lambda J_{\perp \text { eff }}\left[\left(\epsilon_{\perp} \chi_{\|}+\epsilon_{\|} \chi_{\perp}\right) / 2+\epsilon_{\|} \epsilon_{\perp}\left(\chi_{\perp}^{z}+\chi_{\|}^{z}\right)\right], \\
X_{2} & =-\alpha \lambda J_{\perp \text { eff }}\left[\epsilon_{\|} \epsilon_{\perp} \chi_{\|} / 2+\epsilon_{\perp}\left(\chi_{\|}^{z}+C_{\perp}^{z}\right)+\epsilon_{\|} C_{\perp} / 2\right]-\epsilon_{\perp} J_{\perp \text { eff }}^{2} / 2,
\end{aligned}
$$

with the spinon correlation functions $\chi_{\|}=\left\langle S_{a i}^{+} S_{a i+\hat{\eta}}^{-}\right\rangle, \chi_{\|}^{z}=\left\langle S_{a i}^{z} S_{i+\hat{\eta}}^{z}\right\rangle, \chi_{\perp}=\left\langle S_{1 i}^{+} S_{2 i}^{-}\right\rangle, \chi_{\perp}^{z}=\left\langle S_{1 i}^{z} S_{2 i}^{z}\right\rangle, C_{\|}=$ $(1 / 4) \sum_{\hat{\eta} \hat{\eta}^{\prime}}\left\langle S_{a i+\hat{\eta}}^{+} S_{a i+\hat{\eta}^{\prime}}^{-}\right\rangle, C_{\|}^{z}=(1 / 4) \sum_{\hat{\eta} \hat{\eta}^{\prime}}\left\langle S_{a i+\hat{\eta}}^{z} S_{a i+\hat{\eta}^{\prime}}^{z}\right\rangle, C_{\perp}=(1 / 2) \sum_{\hat{\eta}}\left\langle S_{2 i}^{+} S_{1 i+\hat{\eta}}^{-}\right\rangle$, and $C_{\perp}^{z}=(1 / 2) \sum_{\hat{\eta}}\left\langle S_{1 i}^{z} S_{2 i+\hat{\eta}}^{z}\right\rangle$. In order to satisfy the sum rule for the correlation function $\left\langle S_{a i}^{+} S_{a i}^{-}\right\rangle=1 / 2$ in the absence of the antiferromagnetic long-range-order, a decoupling parameter $\alpha$ has been introduced in the mean-field calculation, which can be regarded as the vertex correction ${ }^{19}$. All the mean-field order parameters, decoupling parameter, and chemical potential have been determined self-consistently, as done in the two-dimensional case $\mathrm{e}^{18,19}$.

In Fig. 1, we represent the dynamical spin structure factor $S(\mathbf{k}, \omega)$ in the $(k, \omega)$ plane at doping $p=0.16$ with temperature $T=0.05 J_{\|}$for $t_{\|} / J_{\|}=2.5$, (a) $t_{\perp} / t_{\|}=0.5, J_{\perp} / J_{\|}=0.5$, and (b) $t_{\perp} / t_{\|}=0.7, J_{\perp} / J_{\|}=0.7$, hereafter we use the units of $[2 \pi]$. Obviously, an interchain coupling dependence of the incommensurate-commensurate transition occurs. To check this point explicitly, the calculated dynamical spin structure factor spectrum has been used to extract the doping and interchain coupling dependence of the incommensurability $\delta$, defined as the deviation of the peak position from the antiferromagnetic wave vector $\mathbf{Q}=[1 / 2]$, and the results in $T=0.05 J_{\|}$and $\omega=0.4 J_{\|}$for $t_{\|} / J_{\|}=2.5, t_{\perp} / t_{\|}=0$ and $J_{\perp} / J_{\|}=0$ (dash-dotted line), $t_{\perp} / t_{\|}=0.3$ and $J_{\perp} / J_{\|}=0.3$ (solid line), $t_{\perp} / t_{\|}=0.5$ and $J_{\perp} / J_{\|}=0.5$ (long-dashed line), $t_{\perp} / t_{\|}=0.65$ and $J_{\perp} / J_{\|}=0.65$ (short-dashed line), and $t_{\perp} / t_{\|}=0.69$ and $J_{\perp} / J_{\|}=0.69$ (dotted line) are shown in Fig. 2. We therefore find that there are a critical values of $t_{\perp} / t_{\|}=0.7$ and $J_{\perp} / J_{\|}=0.7$ in $T=0.05 J_{\|}$and $\omega=0.4 J_{\|}$. In the weak coupling regime with $t_{\perp} / t_{\|} \ll 0.7$ and $J_{\perp} / J_{\|} \ll 0.7$, the commensurate scattering peak near the half-filling is split into two peaks at $[(1 \pm \delta) / 2]$, where $\delta$ increases initially with the hole concentration in lower dopings, but it saturates at higher dopings. In this case, spinons and holons are more likely to move along the legs of the ladders, rendering the materials quasi-one-dimension. However, the range of the incommensurate spin correlation decreases with increasing the strength of the interchain coupling, and the commensurate spin fluctuation appears in the whole doping range in the strong coupling regime with $t_{\perp} / t_{\|} \geq$ 0.7 and $J_{\perp} / J_{\|} \geq 0.7$. Many experimental analyses ${ }^{1,5,7}$ have indicated that $J_{\perp} / J_{\|}>0.7$ for the doped two-leg ladder antiferromagnet $\mathrm{Sr}_{14-x} \mathrm{Ca}_{x} \mathrm{Cu}_{24} \mathrm{O}_{41}$, this is why that the incommensurate spin correlation is not observed 
in $\mathrm{Sr}_{14-x} \mathrm{Ca}_{x} \mathrm{Cu}_{24} \mathrm{O}_{41}$ from the inelastic neutron scattering experiments ${ }^{1,5,7}$. For a better understanding of the evolution of the commensurate scattering peak with energy in $\mathrm{Sr}_{14-x} \mathrm{Ca}_{x} \mathrm{Cu}_{24} \mathrm{O}_{41}$, we have made a series of scans for the dynamical spin structure factor $S(\mathbf{k}, \omega)$ at different dopings, and the result for $p=0.2$ with $T=0.05 J_{\|}$for $t_{\|} / J_{\|}=2.5, t_{\perp} / t_{\|}=0.77$ and $J_{\perp} / J_{\|}=0.77$ is plotted in Fig. 3 in comparison with the experimental data ${ }^{7}$ taken on $\mathrm{Sr}_{14-x} \mathrm{Ca}_{x} \mathrm{Cu}_{24} \mathrm{O}_{41}$ (inset) with $x=11.5(p \approx 0.2)$ and $J_{\perp} / J_{\|}=0.77$. This result shows that the commensurate spin fluctuation is energy dependent, and the commensurate scattering peak, which is similar to the resonance peak in the bilayer cuprate in the normal state ${ }^{10}$, is located at energy $\omega=0.93 J_{\|}$. This reflects that the anticipated spin gap $\Delta_{S}=0.93 J_{\|} \approx 83.7 \mathrm{meV}$ (Ref. ${ }^{7}$ ) is larger than the spin gap $\approx 32.1 \mathrm{meV}$ observed ${ }^{7}$ in $\mathrm{Sr}_{14-x} \mathrm{Ca}_{x} \mathrm{Cu}_{24} \mathrm{O}_{41}$, which may mean that the simplest two-leg $t-J$ ladder can not be regarded as the complete model for the quantitative comparison with the doped two-leg ladder antiferromagnet. Furthermore, we have also made a series of scans for the dynamical spin structure factor at different temperatures, and found that the weight of the peak is suppressed severely
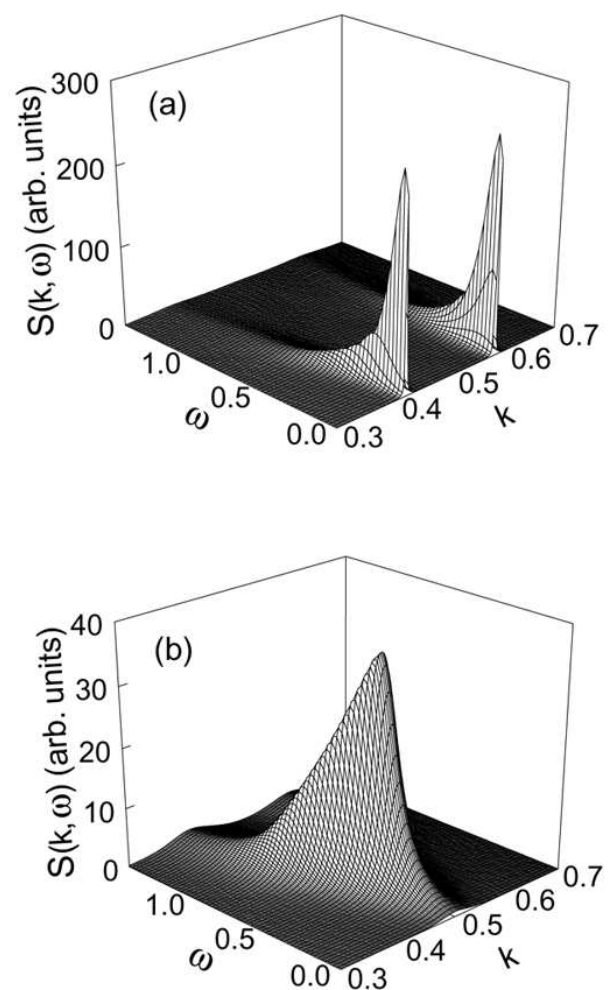

FIG. 1. The dynamical spin structure factor in the $(k, \omega)$ plane at $p=0.16$ with $T=0.05 J_{\|}$for $t_{\|} / J_{\|}=2.5$, (a) $t_{\perp} / t_{\|}=0.5$ and $J_{\perp} / J_{\|}=0.5$, and (b) $t_{\perp} / t_{\|}=0.70$ and $J_{\perp} / J_{\|}=0.70$.

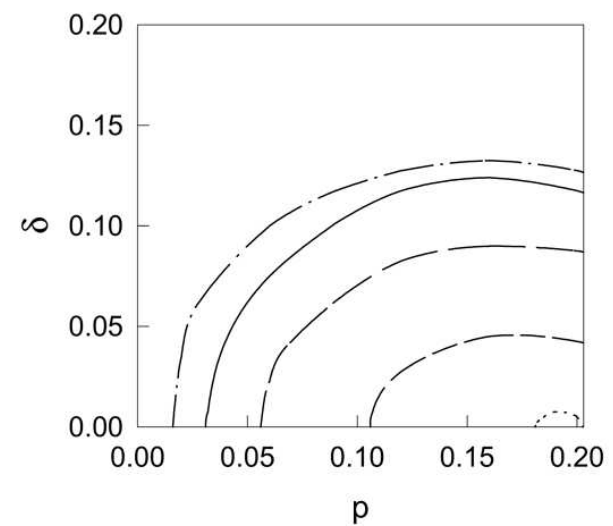

FIG. 2. The doping dependence of incommensurability $\delta(x)$ in $T=0.05 J_{\|}$and $\omega=0.4 J_{\|}$for $t_{\|} / J_{\|}=2.5, t_{\perp} / t_{\|}=0$ and $J_{\perp} / J_{\|}=0$ (dash-dotted line), $t_{\perp} / t_{\|}=0.3$ and $J_{\perp} / J_{\|}=0.3$ (solid line),$t_{\perp} / t_{\|}=0.5$ and $J_{\perp} / J_{\|}=0.5$ (long-dashed line), $t_{\perp} / t_{\|}=0.65$ and $J_{\perp} / J_{\|}=0.65$ (short-dashed line), and $t_{\perp} / t_{\|}=0.69$ and $J_{\perp} / J_{\|}=0.69$ (dotted line). 
with increasing temperatures. Our present results are in qualitative agreement with the experimental data ${ }^{7}$. On the other hand, our present conclusion in the strong coupling regime contradict the numerical result of the isotropic two-leg Hubbard ladder ${ }^{20}$, where the equal-time spin structure factor has been calculated using the density-matrix renormalization group method, and the result shows that the commensurate peak at the half-filling is split into two peaks with dopings. But we want to stress that these results are obtained within the two-leg Hubbard ladder, where the Hubbard $U$ is finite. However, in the $t-J$ model, the doubly occupied Hilbert space has been pushed to infinity as the Hubbard $U \rightarrow \infty$, and therefore the dynamical spin structure factor in the $t$ - $J$ model only describes the lower Hubbard band. This may lead to some different results between the Hubbard and $t$ - $J$ models. Of cause, this has to be checked by the further density-matrix renormalization group study for the $t-J$ ladder.

One of the most important features of the spin dynamics in the doped two-leg ladder antiferromagnet is the spinlattice relaxation time $1 / T_{1}{ }^{8}$. This spin-lattice relaxation time is closely related with the dynamical spin structure factor, and can be expressed as,

$$
1 / T_{1}=2 K_{B} T /\left(g^{2} \mu_{B}^{2} \hbar\right) \lim _{\omega \rightarrow 0}(1 / N) \sum_{k} F_{\alpha}^{2}(\mathbf{k}) \chi^{\prime \prime}(\mathbf{k}, \omega) / \omega,
$$

with $g$ is the $g$ factor, $\mu_{0}$ is the Bohr magneton, $F_{\alpha}(\mathbf{k})$ is the form factors, and dynamical spin susceptibility $\chi^{\prime \prime}(\mathbf{k}, \omega)$ is related to the dynamical spin structure factor by the fluctuation-dissipation theorem as, $\chi^{\prime \prime}(\mathbf{k}, \omega)=\left(1-e^{-\beta \omega}\right) S(\mathbf{k}, \omega)$. The form factors have dimension of energy, and magnitude determined by atomic physics, and $k$ dependence deter-

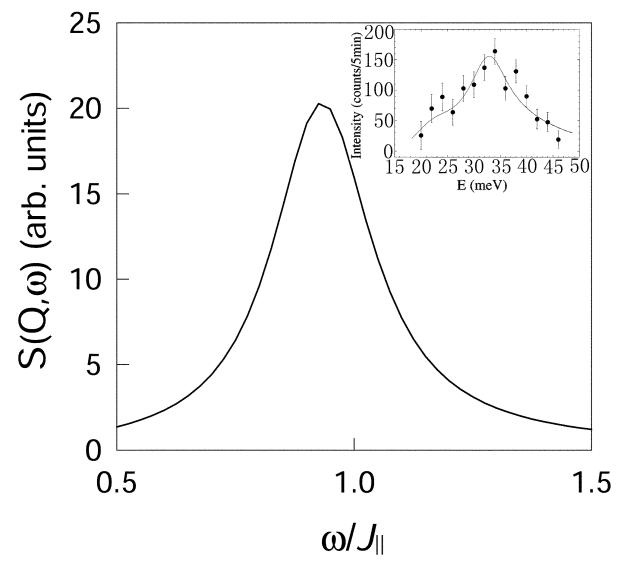

FIG. 3. The dynamical spin structure factor at the AF wave vector $\mathbf{Q}=[1 / 2]$ for $p=0.2$ with $T=0.05 J_{\|}$for $t_{\|} / J_{\|}=2.5$, $t_{\perp} / t_{\|}=0.77$ and $J_{\perp} / J_{\|}=0.77$. Inset: the experimental result ${ }^{7}$ on $\operatorname{Sr}_{14-x} \mathrm{Ca}_{x} \mathrm{Cu}_{24} \mathrm{O}_{41}$ with $x=11.5(p \approx 0.2)$ and $J_{\perp} / J_{\|}=0.77$ taken from Ref. ${ }^{7}$.

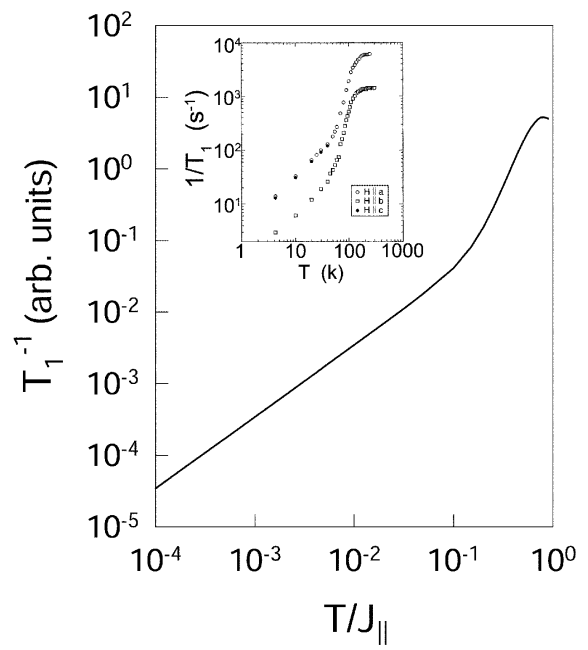

FIG. 4. The temperature dependence of spin-lattice relaxation time $1 / T_{1}$ in both logarithmic scales at $p=0.20$ for $t_{\|} / J_{\|}=2.5, t_{\perp} / t_{\|}=0.77$ and $J_{\perp} / J_{\|}=0.77$. Inset: the experimental result on $\operatorname{Sr}_{14-x} \mathrm{Ca}_{x} \mathrm{Cu}_{24} \mathrm{O}_{41}$ with $x=11.5(p \approx 0.2)$ and $J_{\perp} / J_{\|}=0.77$ taken from Ref. $^{8}$. 
mined by geometry. Since the strong short-range commensurate spin fluctuation in the strong coupling regime in the dynamical spin structure factor (3), the main contribution to $1 / T_{1}$ comes from the region around the antiferromagnetic wave vector, therefore we can set $F_{\alpha}(\mathbf{k})$ as constant without loss the generality ${ }^{13}$. In this case, the spin-lattice relaxation time $1 / T_{1}$ has been evaluated and the result at $p=0.20$ for $t_{\|} / J_{\|}=2.5, t_{\perp} / t_{\|}=0.77$ and $J_{\perp} / J_{\|}=0.77$ is plotted in Fig. 4 in comparison with the experimental data ${ }^{8}$ taken on $\operatorname{Sr}_{14-x} \mathrm{Ca}_{x} \mathrm{Cu}_{24} \mathrm{O}_{41}$ (inset) with $x=11.5$ $(p \approx 0.2)$ and $J_{\perp} / J_{\|}=0.77$, where we have chosen units $\hbar=K_{B}=1$. This result indicates that in low temperatures the spin-lattice relaxation time decreases exponentially with decreasing temperatures, in agreement with the experiments ${ }^{8}$.

The dynamical spin structure factor in Eq. (3) has a well-defined resonance character, where $S(\mathbf{k}, \omega)$ exhibits peaks when the incoming neutron energy $\omega$ is equal to the renormalized spin excitation $E_{k}^{2}=\left(\omega_{k}^{(1)}\right)^{2}+B_{k}^{(1)} \operatorname{Re} \Sigma_{L T}^{(s)}\left(\mathbf{k}, E_{k}\right)$, i.e., $W\left(\mathbf{k}_{c}, \omega\right) \equiv\left[\omega^{2}-\left(\omega_{k_{c}}^{(1)}\right)^{2}-B_{k_{c}}^{(1)} \operatorname{Re} \Sigma_{L T}^{(s)}\left(\mathbf{k}_{c}, \omega\right)\right]^{2}=\left(\omega^{2}-E_{k_{c}}^{2}\right)^{2} \sim 0$ for certain critical wave vectors $\mathbf{k}_{c}$. Then the height of these peaks is determined by the imaginary part of the spinon self-energy $1 / \operatorname{Im} \Sigma_{L T}^{(s)}\left(\mathbf{k}_{c}, \omega\right)$. This renormalized spin excitation is doping, energy, and interchain coupling dependent. In the present spinon selfenergy $\operatorname{Re} \Sigma_{L T}^{(s)}(\mathbf{k}, \omega)=\operatorname{Re} \Sigma_{L}^{(s)}(\mathbf{k}, \omega)+\operatorname{Re} \Sigma_{T}^{(s)}(\mathbf{k}, \omega), \operatorname{Re} \Sigma_{L}^{(s)}(\mathbf{k}, \omega)<0$ favors the one-dimensional behaviors, while $\operatorname{Re} \Sigma_{T}^{(s)}(\mathbf{k}, \omega)>0$ characterizes the quantum interference between the chains in the ladders, therefore there is a competition between $\operatorname{Re} \Sigma_{L}^{(s)}(\mathbf{k}, \omega)$ and $\operatorname{Re} \Sigma_{T}^{(s)}(\mathbf{k}, \omega)$. In the weak coupling regime, the main contribution for $\operatorname{Re} \Sigma_{L T}^{(s)}(\mathbf{k}, \omega)$ may come from $\operatorname{Re} \Sigma_{L}^{(s)}(\mathbf{k}, \omega)$, and spinons and holons are more likely to move along the legs, then the incommensurate spin correlation emerges, where the essential physics is almost the same as in the two-dimensional $t$ - $J$ model ${ }^{18}$. Near the half-filling, the spin excitations are centered around the antiferromagnetic wave vector $[1 / 2]$, so the commensurate antiferromagnetic peak appears there. Upon doping, the holes disturb the antiferromagnetic background. Within the fermion-spin framework, as a result of self-consistent motion of holons and spinons, the incommensurate spin correlation is developed beyond certain critical doping, which means, the low-energy spin excitations drift away from the antiferromagnetic wave vector, where the physics is dominated by the spinon self-energy $\operatorname{Re} \Sigma_{L}^{(s)}(\mathbf{k}, \omega)$ renormalization due to holons. However, the quantum interference effect between the chains manifests itself by the interchain coupling, i.e., this quantum interference increases with increasing the strength of the interchain coupling. Thus in the strong coupling regime, $\operatorname{Re} \Sigma_{T}^{(s)}(\mathbf{k}, \omega)$ may cancel the most incommensurate spin correlation contributions from $\operatorname{Re} \Sigma_{L}^{(s)}(\mathbf{k}, \omega)$, then the commensurate spin fluctuation appears. In this sense, the interchain coupling is a crucial role to determine the symmetry of the spin fluctuation in the doped two-leg ladder antiferromagnet. Since the height of the peaks is determined by damping, it is fully understandable that the weight of the peak is suppressed as the temperature are increased.

To conclude we have discussed the spin dynamics of the doped two-leg ladder antiferromagnet within the $t-J$ ladder. Our calculations clearly show a crossover from the incommensurate spin correlation to commensurate spin fluctuation characterized by the rung to chain coupling. However, in the regions of parameter space given by experiments, the $t-J$ ladder can correctly reproduce all main magnetic features of the doped two-leg ladder antiferromagnet $\mathrm{Sr}_{14-x} \mathrm{Ca}_{x} \mathrm{Cu}_{24} \mathrm{O}_{41}$, including the energy dependence of the neutron scattering peak position and exponential decrease of the nuclear spin-lattice relaxation rate.

\section{ACKNOWLEDGMENTS}

The authors would like to thank Dr. F. Yuan and Professor X. Wang for helpful discussions. This work was supported by the National Natural Science Foundation under the Grant Nos. 10074007, 10125415, and 90103024, the special funds from the Ministry of Science and Technology of China, and the National Science Council under Grant No. NSC 90-2816-M-032-0001-6.

${ }^{1}$ See, e.g., E. Dagotto and T.M. Rice, Science 271, 618 (1996); E. Dagotto, Rep. Prog. Phys. 62, 1525 (1999), and referenes therein.

2 T.M. Rice, S. Gopalan, and M. Sigrist, Europhys. Lett. 23, 445 (1993); E. Dagotto, J. Riera, and D.J. Scalapino, Phys. Rev. B 45, 5744 (1992).

${ }^{3}$ P.W. Anderson, in Frontiers and Borderlines in Many Particle Physics, edited by R.A. Broglia and J.R. Schrieffer (NorthHolland, Amsterdam, 1987), p. 1; Science 235, 1196 (1987). 
${ }^{4}$ M. Uehara, T. Nagata, J. Akimitsu, H. Takahashi, N. Mori, and K. Kinoshita, J. Phys. Soc. Jpn. 65, 2764 (1996).

${ }^{5}$ T. Nagata, M.Uehara, J. Goto, J. Akimitsu, N. Motoyama, H. Eisaki, S. Uchida, H. Takahashi, T. Nakanishi, and N. Mori, Phys. Rev. Lett. 81, 1090 (1998); T. Osafune, N. Motoyama, H. Eisaki, S. Uchida, and S. Tajima, Phys. Rev. Lett. 82, 1313 (1999).

${ }^{6}$ See, e.g., M.A.Kastner, R.J. Birgeneau, G. Shiran, Y. Endoh, Rev. Mod. Phys. 70, 897 (1998), and referenes therein.

${ }^{7}$ S.Katano, T. Nagata, J. Akimitsu, M. Nishi, and K. Kakurai, Phys. Rev. Lett. 82, 636 (1999); M. Matsuda, K. Katsumata, H. Eisaki, N. Motoyama, and S. Uchida, Phys. Rev. B 54, 12199 (1996).

${ }^{8}$ K. Magishi, S. Matsumoto, Y. Kitaoka, K. Ishida, K. Asayama, M. Uehara, T. Nagata, and J. Akimitsu, Phys. Rev. B 57, 11533 (1999); S. Ohsugi, K. Magishi, S. Matsumoto, Y. Kitaoka, T. Nagata, and J. Akimitsu, Phys. Rev. Lett. 82, 4715 (1999).

${ }^{9}$ K. Yamada, C.H. Lee, K. Kurahashi, J. Wada, S. Wakimoto, S. Ueki, H. Kimura, Y. Endoh, S. Hosoya, and G. Shirane, Phys. Rev. B 57, 6165 (1998), and references therein.

${ }^{10}$ P. Dai, H.A. Mook, R.D. Hunt, and F. Dog̃an, Phys. Rev. B63, 54525 (2001), and references therein.

${ }^{11}$ D.V. Khveshchenko and T.M. Rice, Phys. Rev. B50, 252 (1994); D.V. Khveshchenko, Phys. Rev. B50, 380 (1994).

12 M. Sigrist, T.M. Rice, and F.C. Zhang, Phys. Rev. B49, 12058 (1994); D.A. Ivanov and P.A. Lee, Phys. Rev. B57, 2118 (1998).

${ }^{13}$ F. Naef and X. Wang, Phys. Rev. Lett. 84, 1320 (2000); D.A. Ivanov and P.A. Lee, Phys. Rev. B59, 4803 (1999); A.W. Sandvik, E. Dagotto, and D.J. Scalapino, Phys. Rev. B 53, R2934 (1996); M. Troyer, H. Tsunetsugu, and Würtz, Phys. Rev. B50, 13515 (1994).

${ }^{14}$ S. Schdev and K. Damle, Phys. Rev. Lett 78, 943 (1997).

${ }^{15}$ Shiping Feng, Z. B. Su, and L. Yu, Phys. Rev. B 49, 2368 (1994); Shiping Feng and Feng Yuan, in Symposium on the Frontiers of Physics at Millennium, edited by Y.L. Wu and J.P. Hsu (World Scientific, Singapore, 2001), p. 221.

${ }^{16}$ S. Gopalan, T.M. Rice, and M. Sigrist, Phys. Rev. B 49, 8901 (1994); M. Sigrist, T.M. Rice, and F.C. Zhang, Phys. Rev. B 49, 12058 (1994).

${ }^{17}$ L.B. Ioffe and A.I. Larkin, Phys. Rev. B 39, 8988 (1989); see, also, L. Yu, in Recent Progress in Many-Body Theories, edited by T.L. Ainsworth, C.E. Campbell, B.E. Clements, and E. Krotscheck (Plenum, New York, 1992), Vol. 3, p.157.

${ }^{18}$ Feng Yuan, Shiping Feng, Zhao-Bin Su, and Lu Yu, Phys. Rev. B 64, 224505 (2001); Shiping Feng, Feng Yuan, Zhao-Bin $\mathrm{Su}$, and Lu Yu, Phys. Rev. B 66, 064503 (2002).

19 J. Kondo and K. Yamaji, Prog. Theor. Phys. 47, 807 (1972); Shiping Feng and Yun Song, Phys. Rev. B 55, 642 (1997).

${ }^{20}$ R.M. Noack, S.R. White, and D.J. Scalapino, Physica C 270, 281 (1996); R.M. Noack, S.R. White, and D.J. Scalapino, cond-mat/9601047. 